\title{
Weighted Single-Step Genome-Wide Association Study for Growth Traits in Chinese Simmental Beef Cattle
}

\author{
Zhanwei Zhuang ${ }^{1,2}$, Lingyang Xu ${ }^{1}$, Jie Yang ${ }^{2}{ }^{\circledR}$, Huijiang Gao ${ }^{1}$, Lupei Zhang ${ }^{1}{ }^{\circledR}$, Xue Gao ${ }^{1}$, \\ Junya $\mathrm{Li}^{1}$ and Bo Zhu ${ }^{1, *}$ \\ 1 Laboratory of Molecular Biology and Bovine Breeding, Institute of Animal Sciences, Chinese Academy of \\ Agricultural Sciences, Beijing 100193, China; zwzhuang@outlook.com (Z.Z.); xulingyang@163.com (L.X.); \\ gaohuijiang@caas.cn (H.G.); zhanglupei@caas.cn (L.Z.); gaoxue76@126.com (X.G.); lijunya@caas.cn (J.L.) \\ 2 College of Animal Science and National Engineering Research Center for Breeding Swine Industry, \\ South China Agricultural University, Guangzhou 510642, China; jieyang2012@hotmail.com \\ * Correspondence: zhubo@caas.cn
}

Received: 17 December 2019; Accepted: 6 February 2020; Published: 11 February 2020

\begin{abstract}
Improving the genetic process of growth traits is one of the major goals in the beef cattle industry, as it can increase meat production and reduce the cost of raising animals. Although several quantitative trait loci affecting growth traits in beef cattle have been identified, the genetic architecture of these economically important traits remains elusive. This study aims to map single nucleotide polymorphisms (SNPs) and genes associated with birth weight (BW), yearling weight (YW), average daily gain from birth to yearling (BYADG), and body weight at the age of 18 months (18MW) in a Chinese Simmental beef cattle population using a weighted, single-step, genome-wide association study (wssGWAS). Phenotypic and pedigree data from 6022 animals and genotypes from 744 animals (596,297 SNPs) were used for an association analysis. The results showed that 66 genomic windows explained 1.01-20.15\% of the genetic variance for the four examined traits, together with the genes near the top SNP within each window. Furthermore, the identified genomic windows ( $>1 \%)$ explained $50.56 \%, 57.71 \%, 61.78 \%$, and $37.82 \%$ of the genetic variances for BW, YW, BYADG, and 18MW, respectively. Genes with potential functions in muscle development and regulation of cell growth were highlighted as candidates for growth traits in Simmental cattle (SQOR and TBCB for BW, MYH10 for YW, RLF for BYADG, and ARHGAP31 for 18MW). Moreover, we found 40 SNPs that had not previously been identified as being associated with growth traits in cattle. These findings will further advance our understanding of the genetic basis for growth traits and will be useful for the molecular breeding of BW, YW, BYADG, and 18MW in the context of genomic selection in beef cattle.
\end{abstract}

Keywords: Chinese Simmental beef cattle; weighted single-step GWAS; growth traits; SNP

\section{Introduction}

Beef cattle provide a large proportion of the meat consumed by humans throughout the world [1]. Improving the genetic process of growth traits (e.g., body weight and average daily gain) is one of the major goals in the beef cattle breeding industry, as it can increase meat production and reduce the cost of raising animals $[2,3]$. The key to accelerating the progress towards this goal is to genetically select elite cattle and to mine major genes that affect growth traits. A genome-wide association study (GWAS) can detect significant single nucleotide polymorphisms (SNPs) or genomic regions that are associated with economically important traits based on the linkage disequilibrium (LD) between SNPs and possible causative mutations [4]. GWASs have recently been used to identify several quantitative trait loci (QTLs) and genes associated with growth traits in beef cattle [1,3,5]. For instance, Kim et al. [6] used 
602 crossbred cattle of Bos taurus (Angus) and Bos indicus (Brahman) genotyped for 417 microsatellite markers and detected a total of 35 QTLs for growth traits (e.g., birth weight and yearling weight). Buzanskas et al. [7] performed a GWAS in 404 Canchim beef cattle using BovineHD BeadChip and found four SNPs associated with birth weight. Jahuey-Martinez et al. [8] found 18 SNPs located in 13 Bos taurus chromosomes (BTA) and highlighted five genes (TRAF6, CDH11, KLF7, MIR181A-1 and $P R C P$ ) that were associated with growth traits in a population of 855 Charolais beef cattle genotyped for 76,883 SNPs. Although some progress has been made, the genetic architecture of these economically important traits remains poorly understood. Furthermore, the majority of GWASs for growth traits in beef cattle have only used a small sample of genotyped animals and low-density SNP arrays, which has limited the statistical power of the association analysis $[1,8]$. To address this issue, the weighted single-step GWAS (wssGWAS) is preferable for association analysis in Chinese beef cattle, for which large numbers of individuals have phenotypes and pedigrees but fewer are genotyped.

The wssGWAS estimates the SNP effects using genomic estimated breeding values (GEBVs) by solving a blend of pedigrees and SNPs derived matrix $\mathrm{H}$, which was used in weighted single-step genomic best linear unbiased prediction (wssGBLUP). This approach can make full use of genealogical information and phenotypes of genotyped and nongenotyped animals [9]. The weighted single-step approach has been successfully applied to domesticated animals, and has led to the detection of additional QTLs and candidate genes for growth traits in Nellore cattle [3], semen traits in Duroc boar pigs [10], and milk protein composition traits in Chinese Holstein dairy cattle [11]. However, to our knowledge, few of the studies examining growth traits in Simmental beef cattle have used wssGWAS. Therefore, the objective of this study was to identify genomic regions and candidate genes associated with growth traits (birth weight (BW), yearling weight (YW), average daily gain from birth to yearling (BYADG), and body weight at the age of 18 months (18MW)) in Chinese Simmental beef cattle using the wssGWAS approach. In addition, gene enrichment analysis was performed to better understand the biological processes and pathways shared by trait-associated genes.

\section{Materials and Methods}

\subsection{Ethics Statement}

All animals used in the current study were treated following the guidelines for the care and use of experimental animals established by the Ministry of Agriculture and Rural Affairs of China. The ethics committee of the Science Research Department of the Institute of Animal Sciences, Chinese Academy of Agricultural Sciences (CAAS) (Beijing, China) approved this study. The approval ID/permit numbers are SYXK (Beijing) 2008-007 and SYXK (Beijing) 2008-008.

\subsection{Animals, Phenotypes and Pedigree}

The animals used in this study originated from 12 Chinese Simmental beef cattle core farms. These cattle were raised in different regions of China that participated in the national joint beef cattle breeding and genetic improvement program. In brief, a total of 6022 Simmental beef cattle (2878 males and 3144 females) born from 2001 to 2019 were used in this study. Among them, 6022 animals were used in wssGWAS for BW; 3996 individuals were used in wssGWAS for YW and BYADG; 3137 animals were used in wssGWAS for 18MW. Genealogical information was available for all Chinese Simmental beef cattle (both males and females). The animals born from 2018 to 2019 were only used in the BW association analysis because many of these cattle lacked phenotypic records for YW and 18MW. Yearling weight and 18MW of Simmental beef cattle was recorded at about $360 \pm 30$ days and $540 \pm 30$ days of age, respectively. Average daily gain from birth to yearling was calculated by subtracting the birth weight from the yearling weight and dividing by the number of days during this period. For the four traits under study, outliers beyond three standard deviations were removed before the association analysis. 


\subsection{Genotyping and Quality Control}

A total of 744 Chinese Simmental beef cattle was genotyped using Illumina BovineHD BeadChips, which contained 777,962 SNPs. Quality control (QC) procedures were conducted using the PLINK v1.07 software (Boston, MA, USA) [12]. Individuals with call rates $<95 \%$, SNPs with minor allele frequency $<0.05$, call rates $<95 \%$ and SNPs that failed the Hardy-Weinberg equilibrium test $\left(p<10^{-6}\right)$ were removed. In addition, SNPs were also excluded if they were located on the sex chromosomes or had no positional information. After the QC, a final set of 596,297 SNPs for 744 Simmental beef cattle were retained for subsequent analyses.

\subsection{Weighted Single-Step Genome-Wide Association Study}

The wssGBLUP proposed by Wang et al. [9] was utilized to make use of all available phenotypes, pedigree, and genotypes using the BLUPF90 family programs [13]. The RENUMF90 module was used to extract data for phenotypes, pedigrees, and genomic markers in raw file format. The AIREMLF90 module was used to estimate the variance components that were used in BLUPF90 to predict GEBV. The postGSf90 module was used to conduct the wssGWAS. The four traits were analyzed using the same single trait animal model in wssGBLUP as described below:

$$
y=W b+Z a+e
$$

where $y$ represented a vector of phenotypic observations; $b$ was the vector of fixed effects. In this study, sex, year of birth, use types (meat or dual-purpose), and farms were treated as fixed effects for all traits. In addition, age (in days) was included in models for YW, 18MW and BYADG as fixed effects. $a$ was the vector of additive genetic effects and $e$ denoted the residuals; and $W$ and $Z$ were the incidence matrices of $b$ and $a$, respectively. It was assumed that

$$
a \sim N\left(0, H \sigma_{a}^{2}\right)
$$

and

$$
e \sim N\left(0, I \sigma_{e}^{2}\right)
$$

where $\sigma_{a}^{2}$ and $\sigma_{e}^{2}$ were the additive genetic variance and residual variance, respectively. $H$ was a blend of pedigrees and the SNP derived matrix and $I$ denoted the identity matrix. The inverse of matrix $H$ was calculated as follows:

$$
H^{-1}=A^{-1}+\left[\begin{array}{cc}
0 & 0 \\
0 & G^{-1}-A_{22}^{-1}
\end{array}\right]
$$

where $A$ denoted the numerator relationship matrix based on the pedigree for all individuals; $A_{22}$ was the numerator relationship matrix for the genotyped animals; and the $G$ matrix was a genomic relationship matrix that was constructed as described by Vanraden [14]:

$$
G=\frac{Z D Z^{\prime}}{\sum_{i=1}^{\mathrm{M}} 2 p_{i}\left(1-p_{i}\right)}
$$

where $Z$ was an incidence matrix adjusted for allele frequencies, and $D$ denoted a diagonal matrix of weights for SNP variances. M was the number of markers, and $p_{i}$ represented the minor allele frequency of the $i$ th SNP. The SNP effects and weights for wssGWAS were calculated iteratively as follows [9]:

1. In the first iteration, set $t=1$,

$$
\begin{gathered}
D_{(t)}=I \\
G_{(t)}=\lambda Z D_{(t)} Z^{\prime} \\
\lambda=\frac{1}{\sum_{i=1}^{M} 2 p_{i}\left(1-p_{i}\right)}
\end{gathered}
$$


2. Estimate GEBV for all animals using ssGBLUP approach;

3. Compute SNP effects as

$$
\hat{u}_{(t)}=\lambda D_{(t)} Z^{\prime} G_{(t)}^{-1} \hat{a}_{g}
$$

where $\hat{u}_{(t)}$ was a vector of the SNP effects estimation and $\hat{a}_{g}$ was the GEBV of animals that were genotyped;

4. Calculate SNP weights for the next iteration using

$$
d_{i(t=1)}=\hat{u}_{i(t)}^{2} 2 p_{i}\left(1-p_{i}\right)
$$

where $i$ was the $i$ th SNP;

5. Normalize SNP weights to keep the total genetic variance constant as

$$
D_{(t+1)}=\frac{\operatorname{tr}\left(D_{(t)}\right)}{\operatorname{tr}\left(D_{(t+1)}\right)} D_{(t+1)}
$$

6. Calculate $G_{(t+1)}$

$$
G_{(t+1)}=\frac{Z D_{(t+1)} Z^{\prime}}{\sum_{i=1}^{M} 2 p_{i}\left(1-p_{i}\right)}
$$

7. Set $t=t+1$ and loop to step 2 .

In this study, the procedure was run for three iterations as used in Wang et al. [9] and the wssGWAS results were represented by the proportion of genetic variance explained by the windows of 20 successive adjacent SNPs [15]. The percentage of additive genetic variance explained by the $i$ th SNP window was calculated as:

$$
\frac{\operatorname{var}\left(a_{i}\right)}{\sigma_{a}^{2}} \times 100 \%=\frac{\operatorname{var}\left(\sum_{j=i}^{20} z_{j} \hat{u}_{j}\right)}{\sigma_{a}^{2}} \times 100 \%
$$

where $a_{i}$ was the genetic value of the $i$ th window consisting of 20 adjacent SNPs; $\sigma_{a}^{2}$ was the total genetic variance and $z_{j}$ was a vector genotype of the jth SNP for all animals; and $\hat{u}_{j}$ was the SNP effect of the $j$ th SNP within the $i$ th window. Because the windows size is 20 , the proportion of variance assigned to SNP 1 is calculated from SNP 1 to 20, for SNP 2 it goes from 2 to 21 , and so forth. Therefore, the SNP that contributed approximately equally to the 20-adjacent-SNP window was defined as the most important marker (top SNP).

\subsection{Identification of Candidate Genes}

Genomic windows that explained more than $1.0 \%$ of the genetic variance were selected as possible QTL regions associated with growth traits in Chinese Simmental beef cattle. Genes were searched using the Ensembl database [16] based on the SNP position that belonged to the significant genomic windows. In order to better understand the biological processes and pathways shared by these candidate genes, we conducted GO and KEGG enrichment analysis using DAVID bioinformatics resource (version 6.8) [17]. Significantly enriched terms were assessed using Fisher's exact test $(p<0.05)$ and genes involved in biological processes were highlighted [18].

\section{Results and Discussion}

\subsection{Descriptive Statistics and Heritabilities for the Growth Traits}

Descriptive statistics of the observed phenotypes are shown in Table 1 . The coefficients of variation (CV) for BW, YW, BYADG and 18MW were $11.97 \%, 16.36 \%, 18.45 \%$ and $18.61 \%$, respectively. The results 
indicated that substantial phenotypic variation of these four traits exists in the Simmental beef cattle population. The heritability estimates for BW, YW, BYADG and 18MW in Chinese Simmental beef cattle were $0.42,0.24,0.23$, and 0.43 , respectively.

Table 1. Descriptive statistics and variance components of growth traits in Chinese Simmental beef cattle ${ }^{a}$.

\begin{tabular}{ccccccccccc}
\hline Traits & N & Mean & SD & Min & Max & CV (\%) & $\sigma_{a}^{2}$ & $\sigma_{\boldsymbol{e}}^{2}$ & $\sigma_{p}^{2}$ & $h^{2}(\mathrm{SE})$ \\
\hline BW (kg) & 6022 & 44.96 & 5.38 & 29 & 61 & 11.97 & 10.165 & 14.150 & 24.315 & $0.42 \pm 0.03$ \\
YW (kg) & 3996 & 418.21 & 68.37 & 264.94 & 608.65 & 16.36 & 444.650 & 1352.400 & 1797.050 & $0.24 \pm 0.03$ \\
BYADG (kg/d) & 3996 & 1.03 & 0.19 & 0.6 & 1.54 & 18.45 & 0.003 & 0.010 & 0.013 & $0.23 \pm 0.02$ \\
18MW (kg) & 3137 & 587.41 & 109.36 & 376.84 & 905.24 & 18.61 & 1193.400 & 1598.000 & 2791.400 & $0.43 \pm 0.03$ \\
\hline
\end{tabular}

a Number of animals used in the wssGWAS, $\sigma_{a}^{2}=$ genetic variance, $\sigma_{e}^{2}=$ residual variance, $\sigma_{p}^{2}=$ phenotypic variance, $h^{2}=$ heritability.

\subsection{Summary of the wssGWAS Results}

We performed a wssGWAS in Simmental beef cattle populations to map genetic markers and genes associated with BW, YW, BYADG and 18MW. The wssGWAS results were represented by the proportion of genetic variance explained by windows of 20 successive SNPs (Figure 1). Genomic windows that explained more than $1.0 \%$ of the additive genetic variance of the four traits are shown in Tables 2-5, together with the genes near the most important SNPs within each window. In total, 66 nonredundant windows that explained $1.01-20.15 \%$ of the additive genetic variance for the four growth traits were identified. Furthermore, the identified genomic windows explained 50.56\%, 57.71\%, $61.78 \%$, and $37.82 \%$ of the genetic variances for BW, YW, BYADG, and 18MW, respectively.
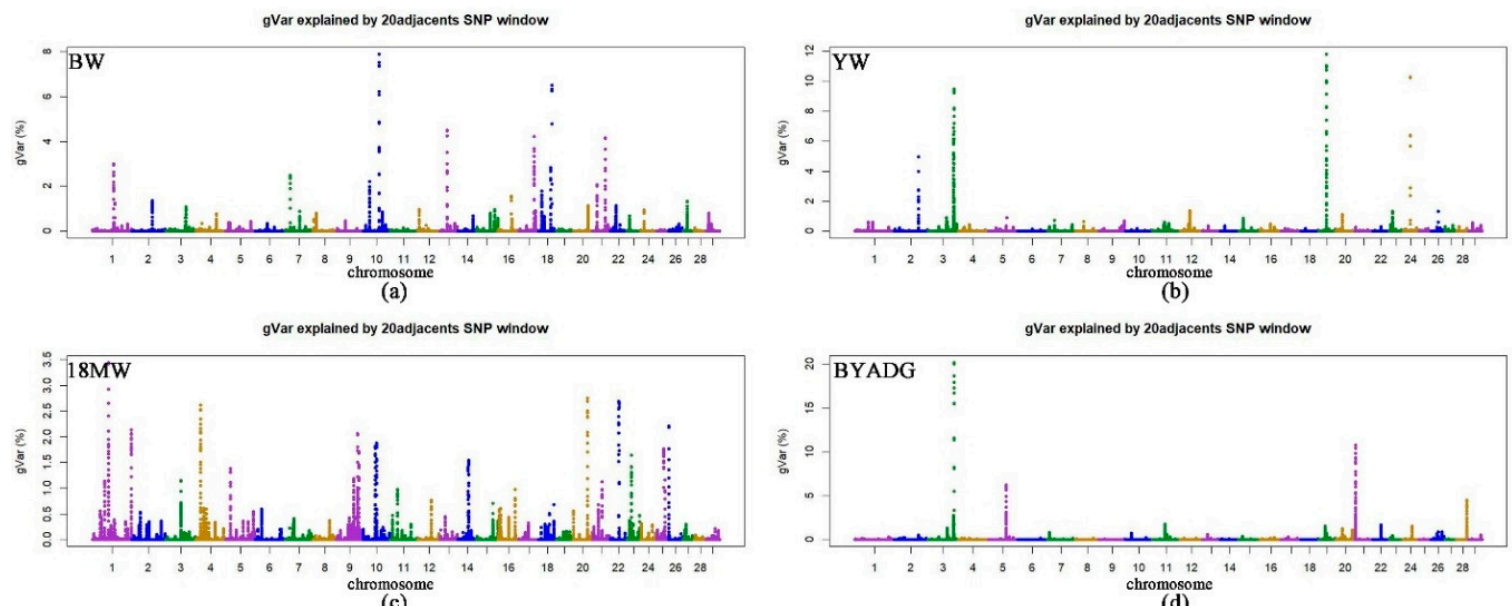

Figure 1. Manhattan plots for the percentage of genetic variance by 20 adjacent SNP windows for growth traits in Chinese Simmental beef cattle. gVar (\%) represent the proportion of genetic variance explained by 20 adjacent SNPs. (a) BW: birth weight; (b) YW: yearling weight; (c) 18MW: body weight at the age of 18 months; (d) BYADG: average daily gain from birth to yearling.

\section{3. wss GWAS for $B W$}

Analysis was undertaken of the association with BW identified 18 genomic windows that were located on BTA1, 2, 3, 7, 10, 13, 16, 17, 18, 20, 21, 22, and 27. The identified genomic windows explained $1.07-7.89 \%$ of the additive genetic variances for BW. Genes nearest to the peak SNPs within each window were treated as potential associated candidates with BW (Table 2). Among these significant windows, the most important region was located at BTA10: 64,843,548-64,888,989 bp, which explained 7.89\% of the genetic variance for BW. The gene adjacent to the top SNP, BovineHD1000018698, was sulfide quinone oxidoreductase $(S Q O R)$. SQOR is a protein coding gene that may interact with the inner mitochondrial membrane in a monotopic fashion and catalyze the mammalian metabolism of $\mathrm{H}_{2} \mathrm{~S}$ 
(hydrogen sulfide) in human [19]. Veeranki and Tyagi et al. [20] proposed a model where $\mathrm{H}_{2} \mathrm{~S}$ may function in skeletal muscle wasting/fibrosis as a result of metabolic complications (such as from obesity), which implied that the features of $\mathrm{H}_{2} \mathrm{~S}$ reversed muscle damage and moderated metabolic myopathy. The second most important window (BTA18: 46,973,033-47,054,361 bp) was located inside the tubulin folding cofactor $\mathrm{B}(T B C B)$ gene, which plays a role in modulating cytoskeletal activity [21]. TBCB was proposed as a candidate gene related to meat quality in pigs due to the correlation between the protein filaments of the cytoskeleton and actin filaments [22]. In the modern beef cattle industry, meat quality traits and growth traits are two important breeding goals in genetic improvement programs. Furthermore, there is a strong genetic correlation between meat quality and growth traits and therefore, the suggestion of $T B C B$ gene as a potential candidate for $\mathrm{BW}$ in cattle is reasonable [23]. BW is a typical polygenic quantitative trait which may be subject to nutritional intake, feeding environment of cows during pregnancy, and in some cases, sex-specific genomic imprinting [24]. Results from this study implied that genetic factors may contribute to a large share of the variation in BW in beef cattle and therefore, the identified SNPs (which explain $>1 \%$ of the genetic variance) can be used for genetic improvement in the context of genomic selection (GS).

Table 2. Windows that explained $>1 \%$ of the additive genetic variance for birth weight in Simmental beef cattle.

\begin{tabular}{|c|c|c|c|c|c|}
\hline $\mathrm{Chr}^{\mathrm{a}}$ & Window Region $(b p)^{b}$ & $g \operatorname{Var}(\%)^{c}$ & topSNP d & Candidate Gene ${ }^{e}$ & Distance \\
\hline 10 & $64,843,548-64,888,989$ & 7.89 & BovineHD1000018698 & SQOR & 195,750 \\
\hline 18 & $46,973,033-47,054,361$ & 6.52 & BovineHD1800013865 & $\widetilde{T B C B}$ & 971 \\
\hline 13 & $32,898,989-32,942,587$ & 4.51 & BovineHD1300009582 & CACNB2 & 106,042 \\
\hline 17 & $59,422,381-59,533,974$ & 4.23 & BovineHD1700016840 & WSB2 & 634 \\
\hline 21 & $54,246,453-54,304,950$ & 4.17 & BovineHD2100015513 & DYNLL1 & 49,145 \\
\hline 1 & $86,864,010-87,000,954$ & 3.00 & BovineHD0100024730 & CCDC39 & 89,435 \\
\hline 18 & $43,486,335-43,539,576$ & 2.83 & BovineHD1800012856 & CEP89 & within \\
\hline 7 & $25,274,362-25,344,621$ & 2.51 & BovineHD0700006961 & CHSY3 & within \\
\hline 10 & $22,200,536-22,227,480$ & 2.20 & BovineHD4100007964 & $T R D C$ & within \\
\hline 21 & $18,442,926-18,507,549$ & 2.06 & BovineHD4100015053 & / & 1 \\
\hline 18 & $9,390,632-9,440,030$ & 1.78 & BovineHD4100013431 & $\mathrm{CDH13}$ & 122,107 \\
\hline 16 & $55,454,686-55,576,782$ & 1.57 & BovineHD1600015438 & BOVAGGRUS & 12,062 \\
\hline 2 & $84,272,058-84,486,086$ & 1.35 & BovineHD0200024049 & SLC39A10 & 461,944 \\
\hline 27 & $17,515,069-17,628,482$ & 1.35 & BovineHD2700005087 & / & / \\
\hline 1 & $93,966,859-94,071,139$ & 1.24 & BovineHD0100026650 & NLGN1 & 334,630 \\
\hline 20 & $58,103,188-58,151,048$ & 1.14 & BovineHD2000016087 & ANKH & 274,075 \\
\hline 22 & $21,743,429-21,782,083$ & 1.14 & BovineHD2200006300 & ITPR1 & within \\
\hline 3 & $83,073,814-83,124,751$ & 1.07 & BovineHD0300023782 & ATG4C & 225,105 \\
\hline
\end{tabular}

${ }^{\mathrm{a}}$ Chromosomes; ${ }^{\mathrm{b}}$ Window position in Ensembl; ${ }^{\mathrm{c}}$ The proportion of the genetic variance explained by 20 adjacent SNP window; ${ }^{\mathrm{d}}$ The SNP that explained the largest proportion of genetic variance within each window (equal to the value of the 20 adjacent SNP window explained); ${ }^{\mathrm{e}}$ Candidate genes near the top SNPs.

\section{4. wssGWAS for $Y W$ and $B Y A D G$}

In total, 14 windows in eight different chromosomes (BTA2, 3, 12, 19, 20, 23, 24 and 26) were associated with $\mathrm{YW}$ (Table 3). The proportion of genetic variance for these windows ranged from $1.11 \%$ to $11.80 \%$. The most significant window (BTA19: $28,728,158-28,766,002 \mathrm{bp}$ ) contributed $11.80 \%$ of the genetic variance of YW. The top SNP (BovineHD1900008433) of this window was located within the myosin heavy chain 10 (MYH10) gene. The MYH10 gene is a member of the myosin superfamily which shares the common features of ATP hydrolysis (ATPase enzyme activity), actin binding, and potential for kinetic energy transduction [25]. Myosin plays an important role in muscle growth and contraction [26,27]. MYH10 is isolated from muscle cells and with functions in contractile, it is also related to myosin in nonmuscle cells [28]. Moreover, Xue et al. [29] found that MYH10 takes part in a pathway related to growth and development in chickens and significantly upregulated the expression pattern at the transcript level. Furthermore, we evaluated the LD pattern of the SNPs in the region around $28.47-28.90 \mathrm{Mb}$ on BTA19. The LD analysis revealed that the region located on 
MYH10 showed a high LD level between the top SNP and nearby SNPs, implying a potential selection signature with regard to YW in Simmental beef cattle (Figure 2a). Therefore, it is reasonable to speculate that $M Y H 10$ is a strong candidate gene for $\mathrm{YW}$ due to its potential roles in the genetic mechanisms of muscle development. For BYADG, 15 windows in nine different chromosomes (BTA3, 5, 11, 19, 20, 21, 22, 24 and 28) were identified (Table 4). Results showed that these windows explained $1.13-20.15 \%$ of the genetic variance for BYADG. The first three most important windows explained approximately $37.14 \%$ of the genetic variance of BYADG in total, which accounted for up to $60 \%$ of the genetic variance of all identified window interpretations. These findings implied that these windows (BTA3: 106,574,782-106,644,015 bp; BTA21: 5,941,998-5,968,820 bp; and BTA5: 77,160,030-77,212,501 bp) need more attention when selecting candidate genes for BYADG.

Table 3. Windows that explained $>1 \%$ of the additive genetic variance for yearling weight in Simmental beef cattle.

\begin{tabular}{|c|c|c|c|c|c|}
\hline $\mathrm{Chr}^{\mathrm{a}}$ & Window Region $(b p)^{b}$ & $\operatorname{gVar}(\%)^{c}$ & topSNPd & Candidate Gene $^{\mathrm{e}}$ & Distance \\
\hline 19 & $28,728,158-28,766,002$ & 11.80 & BovineHD1900008433 & MYH10 & within \\
\hline 24 & $30,102,158-30,164,301$ & 10.30 & BovineHD2400008151 & CHST9 & within \\
\hline 3 & $106,567,276-106,628,358$ & 9.47 & BovineHD0300030609 & $R L F$ & within \\
\hline 3 & $103,471,058-103,518,431$ & 6.91 & BovineHD0300029636 & FAM183A & 27,683 \\
\hline 2 & $98,892,271-98,968,329$ & 4.95 & BovineHD0200028465 & CPS1 & within \\
\hline 3 & $107,082,578-107,188,510$ & 3.09 & BovineHD4100002437 & PABPC4 & 1629 \\
\hline 3 & $105,809,585-105,893,206$ & 1.92 & BovineHD0300030334 & CTPS & within \\
\hline 3 & $105,103,446-105,151,204$ & 1.57 & BovineHD0300030104 & / & / \\
\hline 2 & $98,767,231-98,852,487$ & 1.50 & BovineHD0200028425 & CPS1 & within \\
\hline 12 & $37,317,791-37,375,872$ & 1.36 & BovineHD1200010822 & ATP12A & 395,299 \\
\hline 23 & $13,141,062-13,228,547$ & 1.33 & BovineHD2300003307 & KCNK17 & 19,569 \\
\hline 26 & $28,767,232-28,870,785$ & 1.33 & BovineHD2600007699 & SORCS1 & 377,841 \\
\hline 20 & $23,511,931-23,646,033$ & 1.11 & BovineHD2000007095 & SLC38A9 & within \\
\hline 12 & $37,380,083-37,454,520$ & 1.08 & BovineHD1200010844 & / & / \\
\hline
\end{tabular}

${ }^{a}$ Chromosomes; ${ }^{b}$ Window position in Ensembl; ${ }^{c}$ The proportion of the genetic variance explained by 20 adjacent SNP window; ${ }^{d}$ The SNP explained that the largest proportion of genetic variance within each window (equal to the value of the 20 adjacent SNP window explained); ${ }^{\mathrm{e}}$ Candidate genes near the top SNPs.

Table 4. Windows that explained $>1 \%$ of the additive genetic variance for average daily gain from birth to yearling in Simmental beef cattle.

\begin{tabular}{|c|c|c|c|c|c|}
\hline $\mathrm{Chr}^{\mathrm{a}}$ & Window Region $(b p)^{b}$ & $g \operatorname{Var}(\%)^{c}$ & topSNPd & Candidate Gene ${ }^{e}$ & Distance \\
\hline 3 & $106,574,782-106,644,015$ & 20.15 & BTB-00148396 & $R L F$ & within \\
\hline 21 & $5,941,998-5,968,820$ & 10.77 & BovineHD2100001150 & $A L D H 1 A 3$ & 108,659 \\
\hline 5 & $77,160,030-77,212,501$ & 6.22 & BovineHD0500021921 & / & / \\
\hline 28 & $41,315,052-41,343,055$ & 4.52 & BovineHD2800011614 & WAPAL & 178,111 \\
\hline 5 & $77,289,332-77,341,198$ & 2.90 & BovineHD0500021954 & / & / \\
\hline 3 & $103,471,058-103,518,431$ & 2.79 & BovineHD0300029636 & FAM183A & 27,683 \\
\hline 21 & $6,031,496-6,114,704$ & 2.66 & BovineHD2100001184 & / & / \\
\hline 11 & $47,195,270-47,279,484$ & 1.75 & BovineHD1100013811 & RPIA & 24,890 \\
\hline 22 & $30,689,705-30,734,004$ & 1.69 & BovineHD2200008831 & FOXP1 & within \\
\hline 3 & $105,080,919-105,138,584$ & 1.58 & BovineHD0300030098 & / & l \\
\hline 19 & $24,496,287-24,525,423$ & 1.56 & BovineHD1900007093 & OR1G1 & 9771 \\
\hline 24 & $36,117,632-36,240,233$ & 1.50 & BovineHD2400009926 & ADCYAP1 & within \\
\hline 3 & $79,457,216-79,552,102$ & 1.31 & BovineHD0300022907 & PDE4B & within \\
\hline 20 & $23,511,931-23,646,033$ & 1.26 & BovineHD2000007095 & SLC38A9 & within \\
\hline 20 & $63,943,581-63,965,954$ & 1.13 & BovineHD2000018191 & SEMA5A & 204,352 \\
\hline
\end{tabular}

${ }^{a}$ Chromosomes; ${ }^{b}$ Window position in Ensembl; ${ }^{c}$ The proportion of the genetic variance explained by 20 adjacent SNP window; ${ }^{d}$ The SNP that explained the largest proportion of genetic variance within each window (equal to the value of the 20 adjacent SNP window explained); ${ }^{\mathrm{e}}$ Candidate genes near the top SNPs. 
Table 5. Windows that explained $>1 \%$ of the additive genetic variance for 18 -month weight in Simmental beef cattle.

\begin{tabular}{|c|c|c|c|c|c|}
\hline $\mathrm{Chr}^{\mathrm{a}}$ & Window Region (bp) ${ }^{b}$ & $\operatorname{gVar}(\%)^{c}$ & topSNP d & Candidate Gene ${ }^{e}$ & Distance \\
\hline 1 & $64,788,160-64,867,718$ & 3.44 & BTB-00033090 & ARHGAP31 & within \\
\hline 20 & $55,978,366-56,002,160$ & 2.74 & BovineHD2000015364 & / & / \\
\hline 22 & $32,826,296-32,856,787$ & 2.68 & BovineHD2200009375 & FAM19A4 & within \\
\hline 4 & $18,525,619-18,550,135$ & 2.62 & BovineHD0400005535 & C14H8orf59 & 424,568 \\
\hline 26 & $2,272,143-2,343,667$ & 2.21 & BovineHD2600000342 & / & 1 \\
\hline 1 & $156,048,715-156,119,788$ & 2.13 & BovineHD0100045578 & TBC1D5 & within \\
\hline 9 & $86,926,509-86,984,196$ & 2.06 & BovineHD0900024383 & SASH1 & within \\
\hline 10 & $51,554,432-51,726,000$ & 1.87 & BovineHD1000015443 & FAM63B & within \\
\hline 10 & $48,374,404-48,406,759$ & 1.82 & BovineHD1000014557 & VPS13C & 105,944 \\
\hline 25 & $25,386,519-25,442,136$ & 1.77 & BovineHD2500007181 & KIAA0556 & 29,783 \\
\hline 9 & $93,609,823-93,684,438$ & 1.72 & BovineHD0900026491 & / & / \\
\hline 23 & $19,254,288-19,290,469$ & 1.64 & BovineHD2300004888 & CLIC5 & within \\
\hline 14 & $49,154,187-49,217,544$ & 1.54 & BovineHD1400013993 & MED30 & 195,164 \\
\hline 14 & $47,765,008-47,835,714$ & 1.40 & BovineHD1400013511 & SAMD12 & 14,727 \\
\hline 5 & $18,940,413-19,018,750$ & 1.39 & BovineHD0500005477 & DUSP6 & 336,392 \\
\hline 9 & $71,346,676-71,377,254$ & 1.19 & BovineHD0900019755 & MOXD1 & 4746 \\
\hline 25 & $30,583,072-30,676,658$ & 1.16 & BovineHD2500008480 & / & / \\
\hline 3 & $60,766,989-60,828,010$ & 1.15 & BovineHD0300018250 & TTLL7 & 317,902 \\
\hline 1 & $49,845,978-49,930,370$ & 1.14 & BTB-01076879 & $A L C A M$ & 486,294 \\
\hline 21 & $38,919,896-39,060,313$ & 1.13 & BTB-00818234 & / & / \\
\hline 9 & $88,466,483-88,498,915$ & 1.01 & BovineHD0900024910 & PPP1R14C & within \\
\hline
\end{tabular}

${ }^{\mathrm{a}}$ Chromosomes; ${ }^{\mathrm{b}}$ Window position in Ensembl; ${ }^{\mathrm{c}}$ The proportion of the genetic variance explained by 20 adjacent SNP window; ${ }^{\mathrm{d}}$ The SNP that explained the largest proportion of genetic variance within each window (equal to the value of the 20 adjacent SNP window explained); ${ }^{\mathrm{e}}$ Candidate genes near the top SNPs.

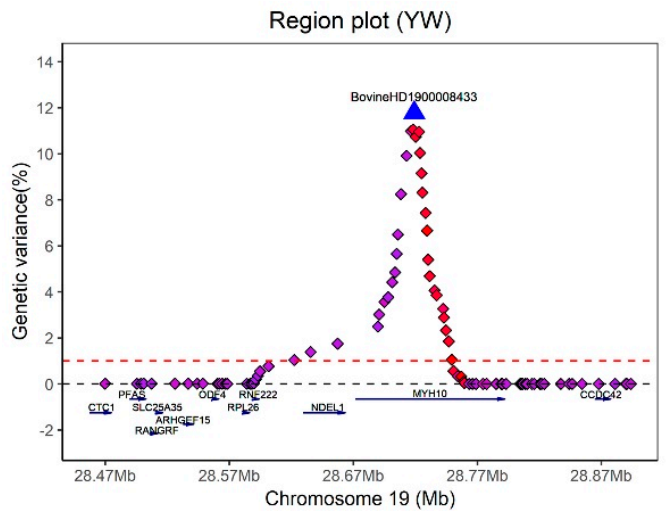

(a)
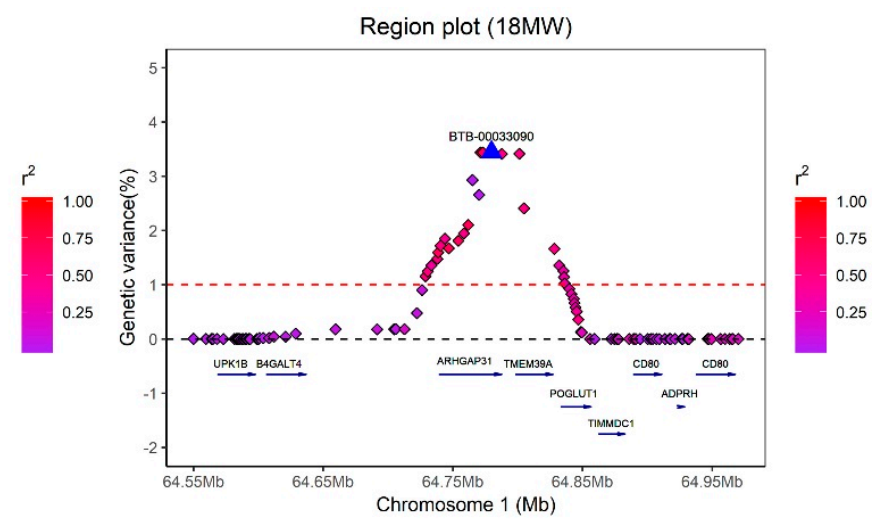

(b)

Figure 2. Region plots of the two major candidate regions on BTA19 and BTA1. Results were shown for YW around $28.47-28.90 \mathrm{Mb}$ on BTA19 (a) and for $18 \mathrm{MW}$ at $64.55-64.98 \mathrm{Mb}$ on BTA1 (b). The primary SNP within each region is denoted by a large blue triangle. Different levels of linkage disequilibrium between the primary SNP and surrounding SNPs were represented by colored rhombi.

Notably, three windows located on BTA3 (103,471,058-103,518,431 bp, 105,080,919-105,138,584 bp, $106,574,782-106,644,015 \mathrm{bp}$ ) were found to be associated with both YW and BYADG, implying a pleiotropic effect for growth traits in beef cattle. Two genes which were adjacent to the top SNP within each window were mined, namely, family with sequence similarity 183 member A (FAM183A) and rearranged L-Myc fusion (RLF). FAM183A has been reported to play a role in autosomal recessive intellectual disability and is expressed in the human brain [30,31]. To our knowledge, few studies have clearly investigated whether FAM183A plays a role in influencing growth traits in domesticated animals and even in the mouse, therefore, further functional studies are required. $R L F$ encodes a Zn- 15 related zinc finger protein and has a general role in transcriptional regulation of fetal and adult tissues in humans [32]. RLF has been reported to play a role in increasing DNA methylation at a number 
of elements related to transcriptional regulation and is involved in maintaining epigenetic marks at CpG island shores and enhancers [33]. DNA methylation plays an essential role in embryonic muscle development and is important for the establishment and maintenance of cellular identity [34,35]. These findings could be helpful for the understanding of mechanisms of muscle development in mammalian animals.

\section{5. wss GWAS for $18 \mathrm{MW}$}

Table 5 shows the 21 windows associated with 18MW which were located on BTA1, 3, 4, 5, 9, 10, $14,20,21,22,23,25$, and 26, together with 16 genes near the most important SNP within each window. The identified genomic windows explained $1.01-3.44 \%$ of the genetic variance for $18 \mathrm{MW}$. The most important window, BTA1: $64788160-64867718 \mathrm{bp}$, contributed to $3.44 \%$ of the genetic variance of 18MW and was located within gene Rho GTPase activating protein 31 (ARHGAP31). ARHGAP31 encodes a GTPase-activating protein (GAP) and plays a role in regulating the cellular processes of cycling between an inactive GDP-bound and active GTP-bound conformation [36]. GAP has been reported to have functions in protein trafficking and cell growth and serves as a molecular switch involved in the regulation of various cytoskeleton-related events and gene transcription [37]. Moreover, LD analysis revealed that a certain level of LD exists between the top SNP (BTB-00033090 within gene ARHGAP31) and its surrounding SNPs in gene transmembrane protein 39A (TMEM39A) (Figure 2b). The potential role of TMEM39A in growth needs further investigation.

\subsection{Potential Genomic Regions and Candidate Genes Reveal the Complexity of the Genetic Architecture of Growth Traits}

In an attempt to better understand the biological processes and pathways shared by the trait-associated genes, we searched 51 genes near the SNPs within each window of the four growth traits. We then performed KEGG and GO enrichment analysis. Three GO terms and no KEGG pathways were enriched for the growth traits analyzed. The enriched GO terms are involved in neuromuscular processes controlling balance (GO: 0050885) consisting of chloride intracellular channel 5 (CLIC5), aldehyde dehydrogenase 1 family member A3 (ALDH1A3), and MYH10 genes; calcium-dependent cell-cell adhesion via plasma membrane cell adhesion molecules (GO: 0016339) consisting of cadherin 13 (CDH13) and neuroligin 1 (NLGN1) genes; motor neuron axon guidance (GO:0008045) including activated leukocyte cell adhesion molecule (ALCAM) and forkhead box P1 (FOXP1) genes. Given the potential roles of the three GO terms in biological processes, their involvement in the growth traits were further analyzed. We searched genes function based on literature reports. Then, the MYH10, CDH13, and FOXP1 genes were highlighted as the main candidates for the growth traits of the three terms, respectively. Notably, MYH10 gene has been highlighted as a strong candidate in the association analysis for YW and additional laboratory functional experiments would be needed. CDH13 gene encodes a member of the cadherin superfamily and acts as a negative regulator of axon growth during neural differentiation [38]. FOXP1 gene plays an important role in the regulation of tissue and cell type-specific gene transcription during both development and adulthood and controls adipocyte differentiation [39]. Results in GO enrichment analyses further extend to suggest that many genes are involved with growth development.

Many studies have reported QTLs and genes associated with growth traits in cattle (e.g., body weight and average daily gain) using the GWAS strategy $[40,41]$. However, few QTLs have consistently been identified as being associated with growth traits among breeds of cattle, including for Brangus heifers [42], Japanese Black (Wagyu) cattle [43], Charolais beef cattle [8], Siberia cattle [1], Nellore cattle [3], and the Chinese Simmental beef cattle examined in this study. These findings imply that further in-depth research is required to determine whether breed-specific QTLs exist. Despite the fact that we have recently uncovered the near-complete genome sequences of several organisms, our knowledge of the genes that underlie phenotypic differences within domestic animals remains rudimentary [44]. In particular, for complex quantitative traits, such as growth traits, the genetic 
basis may be subject to a number of factors including natural selection, inheritance, and evolutionary forces $[45,46]$. The results from our study suggest the complexity of genetic mechanisms of growth traits in Chinese Simmental beef cattle, as numerous potential genomic regions and candidate genes were associated with growth traits. Moreover, to evaluate whether SNPs associated with BW, YW, BYADG and 18MW identified in the present study correspond to any previously known QTLs, we compared the significant SNPs within each window from this study with the SNPs in the cattle QTLdb [47] based on the location of SNPs. The 40 SNPs newly found to be associated with growth traits had not been previously characterized as QTL with regard to growth in cattle (Table S1). These findings will further advance our understanding of the genetic basis for growth traits and will be useful for the molecular breeding of BW, YW, BYADG and 18MW in the context of GS in cattle.

\section{Conclusions}

In conclusion, we identified 66 nonredundant windows which explained $1.01-20.15 \%$ of the additive genetic variance for growth traits in Chinese Simmental beef cattle using the wssGWAS approach. Genes with potential functions in muscle development and regulation of cell growth were highlighted as candidates for growth traits in cattle, such as SQOR and TBCB for BW, MYH10 for YW, RLF for BYADG, and ARHGAP31 for 18MW. Specifically, the identified genomic regions will be useful for the genetic improvement of growth traits by allowing the associated SNPs to be assigned with higher weights in genomic selection.

Supplementary Materials: The following are available online at http://www.mdpi.com/2073-4425/11/2/189/s1, Table S1: Comparative mapping of tag SNPs with previous QTLs reported in the cattle QTL database (as of 24 November 2019) and previous GWAS results.

Author Contributions: J.L. and B.Z. conceived and designed the experiments. Z.Z., L.X., J.Y., H.G., L.Z. and X.G. collected the samples and recorded the phenotypes. Z.Z., L.X. and J.Y. analyzed the data. Z.Z. and J.Y. wrote the manuscript. J.L. and B.Z. revised the manuscript. All authors have read and agreed to the published version of the manuscript.

Funding: This research was supported by the National Natural Science Foundation of China (31802049, 31372294 and 31201782), the National Beef Cattle and Yak Industrial Technology System(CARS-37), the Chinese Academy of Agricultural Sciences of Technology Innovation Project (CAAS-XTCX2016010, CAAS-ZDXT2018006 and ASTIP-IAS03), the Cattle Breeding Innovative Research Team of the Chinese Academy of Agricultural Sciences (cxgc-ias-03 and Y2016PT17), the Beijing Natural Science Foundation (6154032), Project of College Innovation Improvement under Beijing Municipality (PXM2016_014207_000012).

Acknowledgments: The authors would like to thank all staffs at the beef cattle core breeding farms for their help in sample collection.

Conflicts of Interest: The authors declare no conflicts of interest. The funders had no role in the design of the study; in the collection, analyses, or interpretation of data; in the writing of the manuscript, or in the decision to publish the results.

\section{References}

1. Igoshin, A.V.; Yudin, N.S.; Belonogova, N.M.; Larkin, D.M. Genome-wide association study for body weight in cattle populations from Siberia. Anim. Genet. 2019, 50, 250-253. [CrossRef] [PubMed]

2. Takeda, M.; Uemoto, Y.; Inoue, K.; Ogino, A.; Nozaki, T.; Kurogi, K.; Yasumori, T.; Satoh, M. Evaluation of feed efficiency traits for genetic improvement in Japanese Black cattle. J. Anim. Sci. 2018, 96, 797-805. [CrossRef] [PubMed]

3. Terakado, A.P.N.; Costa, R.B.; de Camargo, G.M.F.; Irano, N.; Bresolin, T.; Takada, L.; Carvalho, C.V.D.; Oliveira, H.N.; Carvalheiro, R.; Baldi, F.; et al. Genome-wide association study for growth traits in Nelore cattle. Animal 2018, 12, 1358-1362. [CrossRef] [PubMed]

4. Tam, V.; Patel, N.; Turcotte, M.; Bosse, Y.; Pare, G.; Meyre, D. Benefits and limitations of genome-wide association studies. Nat. Rev. Genet. 2019, 20, 467-484. [CrossRef]

5. Seabury, C.M.; Oldeschulte, D.L.; Saatchi, M.; Beever, J.E.; Decker, J.E.; Halley, Y.A.; Bhattarai, E.K.; Molaei, M.; Freetly, H.C.; Hansen, S.L.; et al. Genome-wide association study for feed efficiency and growth traits in U.S. beef cattle. BMC Genom. 2017, 18, 386. [CrossRef] 
6. Kim, J.J.; Farnir, F.; Savell, J.; Taylor, J.F. Detection of quantitative trait loci for growth and beef carcass fatness traits in a cross between Bos taurus (Angus) and Bos indicus (Brahman) cattle. J. Anim. Sci. 2003, 81, 1933-1942. [CrossRef]

7. Buzanskas, M.E.; Grossi, D.A.; Ventura, R.V.; Schenkel, F.S.; Sargolzaei, M.; Meirelles, S.L.; Mokry, F.B.; Higa, R.H.; Mudadu, M.A.; da Silva, M.V.; et al. Genome-wide association for growth traits in Canchim beef cattle. PLoS ONE 2014, 9, e94802. [CrossRef]

8. Jahuey-Martinez, F.J.; Parra-Bracamonte, G.M.; Sifuentes-Rincon, A.M.; Martinez-Gonzalez, J.C.; Gondro, C.; Garcia-Perez, C.A.; Lopez-Bustamante, L.A. Genomewide association analysis of growth traits in Charolais beef cattle. J. Anim. Sci. 2016, 94, 4570-4582. [CrossRef]

9. Wang, H.; Misztal, I.; Aguilar, I.; Legarra, A.; Muir, W.M. Genome-wide association mapping including phenotypes from relatives without genotypes. Genet. Res. (Camb) 2012, 94, 73-83. [CrossRef]

10. Marques, D.B.D.; Bastiaansen, J.W.M.; Broekhuijse, M.; Lopes, M.S.; Knol, E.F.; Harlizius, B.; Guimaraes, S.E.F.; Silva, F.F.; Lopes, P.S. Weighted single-step GWAS and gene network analysis reveal new candidate genes for semen traits in pigs. Genet. Sel. Evol. 2018, 50, 40. [CrossRef]

11. Zhou, C.; Li, C.; Cai, W.; Liu, S.; Yin, H.; Shi, S.; Zhang, Q.; Zhang, S. Genome-Wide Association Study for Milk Protein Composition Traits in a Chinese Holstein Population Using a Single-Step Approach. Front. Genet. 2019, 10, 72. [CrossRef] [PubMed]

12. Purcell, S.; Neale, B.; Todd-Brown, K.; Thomas, L.; Ferreira, M.A.; Bender, D.; Maller, J.; Sklar, P.; de Bakker, P.I.; Daly, M.J.; et al. PLINK: A tool set for whole-genome association and population-based linkage analyses. Am. J. Hum. Genet. 2007, 81, 559-575. [CrossRef] [PubMed]

13. Misztal, I.; Tsuruta, S.; Lourenco, D.A.L.; Masuda, Y.; Aguilar, I.; Legarra, A.; Vitezica, Z. Manual for BLUPF90 Family Programs. Available online: http://nce.ads.uga.edu/wiki/doku.php?id=documentation (accessed on 11 November 2019).

14. VanRaden, P.M. Efficient methods to compute genomic predictions. J. Dairy Sci. 2008, 91, 4414-4423. [CrossRef] [PubMed]

15. Zhang, X.; Lourenco, D.; Aguilar, I.; Legarra, A.; Misztal, I. Weighting Strategies for Single-Step Genomic BLUP: An Iterative Approach for Accurate Calculation of GEBV and GWAS. Front. Genet. 2016, 7, 151. [CrossRef] [PubMed]

16. Ensembl Database. Assemble: UMD3.1. Available online: http://oct2018.archive.ensembl.org/Bos_taurus/ Info/Index (accessed on 13 November 2019).

17. DAVID Bioinformatics Resource. Available online: https://david.ncifcrf.gov (accessed on 13 November 2019).

18. Zhuang, Z.; Li, S.; Ding, R.; Yang, M.; Zheng, E.; Yang, H.; Gu, T.; Xu, Z.; Cai, G.; Wu, Z.; et al. Meta-analysis of genome-wide association studies for loin muscle area and loin muscle depth in two Duroc pig populations. PLoS ONE 2019, 14, e0218263. [CrossRef]

19. Jackson, M.R.; Melideo, S.L.; Jorns, M.S. Role of human sulfide: Quinone oxidoreductase in H2S metabolism. Methods Enzymol. 2015, 554, 255-270.

20. Veeranki, S.; Tyagi, S.C. Role of hydrogen sulfide in skeletal muscle biology and metabolism. Nitric. Oxide 2015, 46, 66-71. [CrossRef]

21. Watanabe, T.K.; Shimizu, F.; Nagata, M.; Kawai, A.; Fujiwara, T.; Nakamura, Y.; Takahashi, E.; Hirai, Y. Cloning, expression, and mapping of CKAPI, which encodes a putative cytoskeleton-associated protein containing a CAP-GLY domain. Cytogenet. Cell Genet. 1996, 72, 208-211. [CrossRef]

22. Ponsuksili, S.; Murani, E.; Trakooljul, N.; Schwerin, M.; Wimmers, K. Discovery of candidate genes for muscle traits based on GWAS supported by eQTL-analysis. Int. J. Biol. Sci. 2014, 10, 327-337. [CrossRef]

23. Mudadu, M.A.; Porto-Neto, L.R.; Mokry, F.B.; Tizioto, P.C.; Oliveira, P.S.; Tullio, R.R.; Nassu, R.T.; Niciura, S.C.; Tholon, P.; Alencar, M.M.; et al. Genomic structure and marker-derived gene networks for growth and meat quality traits of Brazilian Nelore beef cattle. BMC Genom. 2016, 17, 235.

24. Dillon, J.A.; Riley, D.G.; Herring, A.D.; Sanders, J.O.; Thallman, R.M. Genetic effects on birth weight in reciprocal Brahman-Simmental crossbred calves. J. Anim. Sci. 2015, 93, 553-561. [CrossRef] [PubMed]

25. Simons, M.; Wang, M.; McBride, O.W.; Kawamoto, S.; Yamakawa, K.; Gdula, D.; Adelstein, R.S.; Weir, L. Human nonmuscle myosin heavy chains are encoded by two genes located on different chromosomes. Circ. Res. 1991, 69, 530-539. [CrossRef] [PubMed]

26. Baldwin, K.M.; Haddad, F. Effects of different activity and inactivity paradigms on myosin heavy chain gene expression in striated muscle. J. Appl.Physiol. (Bethesda Md. 1985) 2001, 90, 345-357. [CrossRef] [PubMed] 
27. Glasheen, B.M.; Ramanath, S.; Patel, M.; Sheppard, D.; Puthawala, J.T.; Riley, L.A.; Swank, D.M. Five Alternative Myosin Converter Domains Influence Muscle Power, Stretch Activation, and Kinetics. Biophys J. 2018, 114, 1142-1152. [CrossRef]

28. Yang, F.; Wei, Q.; Adelstein, R.S.; Wang, P.J. Non-muscle myosin IIB is essential for cytokinesis during male meiotic cell divisions. Dev. Biol. 2012, 369, 356-361. [CrossRef]

29. Xue, Q.; Zhang, G.; Li, T.; Ling, J.; Zhang, X.; Wang, J. Transcriptomic profile of leg muscle during early growth in chicken. PLoS ONE 2017, 12, e0173824. [CrossRef]

30. McSherry, M.; Masih, K.E.; Elcioglu, N.H.; Celik, P.; Balci, O.; Cengiz, F.B.; Nunez, D.; Sineni, C.J.; Seyhan, S.; Kocaoglu, D.; et al. Identification of candidate gene FAM183A and novel pathogenic variants in known genes: High genetic heterogeneity for autosomal recessive intellectual disability. PLoS ONE 2018, 13, e0208324. [CrossRef]

31. Abugessaisa, I.; Shimoji, H.; Sahin, S.; Kondo, A.; Harshbarger, J.; Lizio, M.; Hayashizaki, Y.; Carninci, P.; Forrest, A.; Kasukawa, T.; et al. FANTOM5 transcriptome catalog of cellular states based on Semantic MediaWiki. Database J. Biol. Databases Curation 2016, baw105. [CrossRef]

32. Makela, T.P.; Hellsten, E.; Vesa, J.; Hirvonen, H.; Palotie, A.; Peltonen, L.; Alitalo, K. The rearranged L-myc fusion gene (RLF) encodes a Zn-15 related zinc finger protein. Oncogene 1995, 11, 2699-2704.

33. Harten, S.K.; Oey, H.; Bourke, L.M.; Bharti, V.; Isbel, L.; Daxinger, L.; Faou, P.; Robertson, N.; Matthews, J.M.; Whitelaw, E. The recently identified modifier of murine metastable epialleles, Rearranged L-Myc Fusion, is involved in maintaining epigenetic marks at CpG island shores and enhancers. BMC Biol. 2015, 13, 21. [CrossRef]

34. Liu, Z.; Han, S.; Shen, X.; Wang, Y.; Cui, C.; He, H.; Chen, Y.; Zhao, J.; Li, D.; Zhu, Q.; et al. The landscape of DNA methylation associated with the transcriptomic network in layers and broilers generates insight into embryonic muscle development in chicken. Int. J. Biol. Sci. 2019, 15, 1404-1418. [CrossRef] [PubMed]

35. Carrio, E.; Suelves, M. DNA methylation dynamics in muscle development and disease. Front. Aging Neurosci. 2015, 7, 19. [CrossRef] [PubMed]

36. Caron, C.; DeGeer, J.; Fournier, P.; Duquette, P.M.; Luangrath, V.; Ishii, H.; Karimzadeh, F.; Lamarche-Vane, N.; Royal, I. CdGAP/ARHGAP31, a Cdc42/Rac1 GTPase regulator, is critical for vascular development and VEGF-mediated angiogenesis. Sci. Rep. UK 2016, 6, 27485. [CrossRef]

37. Moon, S.Y.; Zheng, Y. Rho GTPase-activating proteins in cell regulation. Trends Cell Biol. 2003, 13, 13-22. [CrossRef]

38. Forero, A.; Rivero, O.; Wäldchen, S.; Ku, H.-P.; Kiser, D.P.; Gärtner, Y.; Pennington, L.S.; Waider, J.; Gaspar, P.; Jansch, C. Cadherin-13 Deficiency Increases Dorsal Raphe 5-HT Neuron Density and Prefrontal Cortex Innervation in the Mouse Brain. Front. Cell Neurosci. 2017, 11, 307. [CrossRef] [PubMed]

39. Liu, P.; Huang, S.; Ling, S.; Xu, S.; Wang, F.; Zhang, W.; Zhou, R.; He, L.; Xia, X.; Yao, Z.; et al. Foxp1 controls brown/beige adipocyte differentiation and thermogenesis through regulating $\beta 3$-AR desensitization. Nat. Commun. 2019, 10, 5070. [CrossRef]

40. Yin, T.; Konig, S. Genome-wide associations and detection of potential candidate genes for direct genetic and maternal genetic effects influencing dairy cattle body weight at different ages. Genet. Sel. Evol. 2019, 51, 4. [CrossRef]

41. Santana, M.H.; Utsunomiya, Y.T.; Neves, H.H.; Gomes, R.C.; Garcia, J.F.; Fukumasu, H.; Silva, S.L.; Leme, P.R.; Coutinho, L.L.; Eler, J.P.; et al. Genome-wide association study for feedlot average daily gain in Nellore cattle (Bos indicus). J. Anim. Breed Genet. 2014, 131, 210-216. [CrossRef]

42. Peters, S.O.; Kizilkaya, K.; Garrick, D.J.; Fernando, R.L.; Reecy, J.M.; Weaber, R.L.; Silver, G.A.; Thomas, M.G. Bayesian genome-wide association analysis of growth and yearling ultrasound measures of carcass traits in Brangus heifers. J. Anim. Sci. 2012, 90, 3398-3409. [CrossRef]

43. Mizoshita, K.; Watanabe, T.; Hayashi, H.; Kubota, C.; Yamakuchi, H.; Todoroki, J.; Sugimoto, Y. Quantitative trait loci analysis for growth and carcass traits in a half-sib family of purebred Japanese Black (Wagyu) cattle. J. Anim. Sci. 2004, 82, 3415-3420. [CrossRef]

44. Andersson, L.; Georges, M. Domestic-animal genomics: Deciphering the genetics of complex traits. Nat. Rev. Genet. 2004, 5, 202-212. [CrossRef] [PubMed] 
45. Povysil, G.; Petrovski, S.; Hostyk, J.; Aggarwal, V.; Allen, A.S.; Goldstein, D.B. Rare-variant collapsing analyses for complex traits: Guidelines and applications. Nat. Rev. Genet. 2019. [CrossRef] [PubMed]

46. Barton, N.H.; Keightley, P.D. Understanding quantitative genetic variation. Nat. Rev. Genet. 2002, 3, 11-21. [CrossRef] [PubMed]

47. Animal QTL Database. Available online: https://www.animalgenome.org/cgi-bin/QTLdb/BT/index (accessed on 24 November 2019).

(c)

(C) 2020 by the authors. Licensee MDPI, Basel, Switzerland. This article is an open access article distributed under the terms and conditions of the Creative Commons Attribution (CC BY) license (http://creativecommons.org/licenses/by/4.0/). 